\title{
Profit-Maximizing Strategies for an Artificial Payment Card Market. Is Learning Possible?
}

\author{
Biliana Alexandrova-Kabadjova ${ }^{1}$, Edward Tsang ${ }^{2}$, Andreas Krause ${ }^{3}$ \\ ${ }^{1}$ General Directorate of Payment Systems and Risks, Bank of Mexico, Mexico City, Mexico; ${ }^{2}$ Centre for Computational Finance and \\ Economic Agents (CCFEA), University of Essex, Colchester, England; ${ }^{3}$ School of Management, University of Bath, Bath, England. \\ Email: balexandrova@banxico.org.mx
}

Received July $10^{\text {th }}, 2010$; revised October $1^{\text {st }}, 2010$; accepted December $1^{\text {st }}, 2010$.

\begin{abstract}
In this paper, we study the dynamics of competition in the payment card market. This is done through a multi-agent based model, which captures explicitly the commercial transactions at the point of sale between consumers and merchants. Through simulation, we attempt to model the demand for payment instruments on both sides of the market. Constrained by this complex demand, a Generalised Population Based Incremental Learning (GPBIL) algorithm is applied to find a profit-maximizing strategy, which in addition has to achieve an average number of card transactions. In the present study we compare the performance of a profit-maximizing strategies obtained by the GPBIL algorithm versus the performance of randomly selected strategies. We found that under the search criteria used, GPBIL was capable of improving the price structure and price level over randomly selected strategies.
\end{abstract}

Keywords: Competition in Payment Card markets, Multi-Agent Modeling, Evolutionary Computation

\section{Introduction}

The payment cards, known as credit and debit cards, in the last two decades have become an important element of the modern economies. The growing importance of these electronic payment instruments is among the reasons why economists and policymakers have put a lot of efforts to understand the way in which the payment card market works [1-4]. This is built over so called two-sided platform [5], in which for a successful transaction with an electronic instrument the consumers have to hold a card and the merchants have to accept it as a payment. Consumers and merchants prefer the use of plastic cards over cash for security reasons or because it could save them time at the counter. These, so called convenience externalities arise among the different retail payment methods used. Further, the higher the number of establishments that accept a particular card, the higher the benefits to the card holders. Similarly, the merchants obtain higher benefits if the number of consumers using a particular card increases. These, known as network externalities, are crucial element of the competition among payment card purveyors.

Platform operators, as Visa and Mastercard, organize their business in a four party scheme: consumers, merchants, issuers (banks that provide cards to the consum- ers) and acquirers (financial institutions that provide electronic terminals to merchants). Each network establishes a specific level of interchange fees, which is charged per transaction and usually flows from acquirers to issuers. For a long time, the main focus of the literature has been on the fee structure of payment cards, with the emphasis laid on the interchange fee $[6,7]$. The research in the field can be divided into models studying the problematic of a single card [1,2,8,9] and [10], and models that allow the competition between payment card platforms [11-14].

Nevertheless, given that the possibility to incorporate the complex market dynamics into an analytical model is limited, the results of the theoretical studies strongly depend on the assumptions regarding the relationships among the market participants. In addition, these models are unable to incorporate the heterogeneity in the preferences of consumers and merchants, neither the externalities arising from the complex dynamics of two-sided demand for electronic payment cards.

Following a different approach, in order to gain better insight of the market, in [15] the authors have developed an agent-based model, which was motivated from [8]. The model simulates the interactions at the point of sale among consumers and merchants. This artificial market 
reproduces the demand of payment cards on both sides and opens the possibilities to study the market dynamics, using computational environment. Further, this work was extended in [16], in which evolutionary computational techniques were applied to find a profit-maximizing strategy. In that environment, instead of representing explicitly issuers and acquirers, the model captures the competition among different platform operators, which payment cards are not compatible. More specifically, each competitor decides his own price level and structure as well as the amount to spend in publicity. The price structure consists in variable and fixed fees on both sides of the market ${ }^{1}$, but each card provider decides which particular fees to apply. The four elements that determined the price, together with the publicity cost form the payment card purveyor's strategy, which is modelled as a vector. Given the complex domain of this vector, there exist multiple possibilities of sampling it. For instances, the authors applied a Generalised Population Based Incremental Learning (GPBIL) [17] algorithm in order to obtain profit-maximizing strategies, which in addition have to achieve an average number of card transactions.

Under this line of research, here we present a study that tests how efficient the GPBIL-strategies reported in [17] are. At this point the Box's statement: "all models are wrong, but some are useful" (p.424 in [20]) could not be more appropriate. Motivated by Box, our aim is to find out if this model is purposeful? To this end, we subject the five elements of a card provider's strategy to training. These are the variable and fixed fees on both sides of the market and the publicity cost. The simplest mechanism of sampling the strategy is to follow a random selection. Alternatively, an instantiation of the vector could be the result of an extensive search over the strategy space constrained by the complex shape of the modelled demand. The space of possible strategies is enormous, due to combinatorial explosion. To improve search efficiency, this search has to be guided by particular criteria of interest, e.g. to find profit-maximizing strategies. The search method chosen could make substantial difference in finding better strategies, in terms of both the quality of the strategies found and search efficiency [21]. In order to find the advantage and disadvantage of using the GPBIL, in the present study our aim is to compare the performance of the GPBIL-strategy and the randomly generated strategy. To that end, we have created an artificial market with nine competitors, in which local interactions among consumers and merchants are reproduced. We report the performance of five

\footnotetext{
${ }^{1}$ The variable fees are charged for each transactions paid with a card, whereas fixed fees are membership fees, which are charged regularly regardless the use of the card.
}

different GPBIL-strategies against ten different random strategies. The test is organized in five exercises, structured in the following way. Each exercise consists of ten independent instantiations of the model, also refereed as cases. In each independent case, eight of the nine competitors use the same profit-maximizing strategy, whereas one competitor applies a randomly generated strategy. Therefore, the exercise consists in comparing the performance of one GPBIL-strategy against ten different random strategies. For each exercise we compare the strategies in two aspects: profit and number of card transactions. The randomly generated strategy needs to obtain higher number of card transactions and better profit in comparison to the GPBIL-strategies, in order for the former to be considered more efficient.

The paper is organized as following: in the next section we briefly introduce the elements' definition and the decisions of the agents interacting at the artificial payment card market. Following, in Section 3 we present the insides of the GPBIL algorithm, whereas in Section 4 we explain the structure of the experiment. Next, Section 5 is dedicated to present the results of the experiment with our observations and finally in Section 6 the related conclusions and suggestions for future work are given.

\section{Artificial Payment Card Market}

The artificial payment card market is a model that simulates commercial transactions among consumers and merchants, following as a framework the model presented in [8]. In this section we introduce formally the elements' definitions and the decisions of the agents.

\subsection{Elements’ Definition}

\subsubsection{Merchants}

Suppose we have a set of merchants $\mathcal{M}$ with $|\mathcal{M}|=N_{\mathcal{M}}$, who are offering a homogeneous good at a common price and face marginal cost of production lower than this price. In other words, we eliminate the price competition among merchants in order to concentrate our analysis on the competition among payment cards. The merchants are located at random intersections of a $N \times N$ lattice, where $N^{2} \gg N_{\mathcal{M}}$. Let the top and bottom edges as well as the right and left edges of this lattice be connected.

\subsubsection{Consumers}

Consumers occupy all remaining intersections of the $N \times$ $N$ lattice. The set of consumers is denoted $C$ with $|C|=N_{C}$, where $N_{C} \gg N_{\mathcal{M}}$ and $N^{2}=N_{C}+N_{\mathcal{M}}$. Each consumer has a budget constraint that allows him to buy in a single interaction exactly one unit of the good offered by the merchants. The utility gained from the consumption of this good is bigger than its price. In order to 
obtain the good any consumer $c \in C$ has to travel to a merchant $m \in \mathcal{M}$. The distance is measured by the Manhattan distance $d_{\mathrm{c}, \mathrm{m}}$ between the locations on the lattice and it imposes travel costs on consumers. The longitude between two adjacent intersections is normalized to unity. Let $\mathcal{M}_{c}$ denotes the set of merchants a consumer considers to go to, given that we restrict him to the nearest merchants ${ }^{2}$.

\subsubsection{Payment Cards}

There exists a set of payment methods $\mathcal{P}$ with $|\mathcal{P}|=N_{\mathcal{P}}+1$ and $N_{\mathcal{P}} \ll N_{\mathcal{M}}$. All payment forms are card payments offered by network operators, with the exception of the first element of the set, which is the benchmark and can be interpreted as cash payment method. The cash is used by all consumers and is accepted by all merchants. Furthermore, in order for a card payment to occur, the consumer as well as the merchant must have a subscription to the card in question. We assume that consumers prefer card payments over cash payments.

A fixed subscription fee of $F_{p} \geq 0$ could be charged per each transaction to the consumer, whereas $\Gamma_{p} \geq 0$ could be charged per each transaction to the merchant. The domains of those fees, $\mathbb{D}_{F_{p}}$ and $\mathbb{D}_{\Gamma_{p}}$ are subsets of real numbers. Cash payments do not attract any fees. For each unit of goods sold using a payment card $p \in \mathcal{P}_{m}$, a merchant $m \in \mathcal{M}$ receives net benefits of $\beta_{p}$. Such benefits may include reduced costs from cash handling and could differ across payment methods. These are identical for all merchants for a given card. The domain $\mathbb{D}_{\beta_{p}}$ is a subset of real numbers. Note that the benefits $\beta_{p}$ could have a negative value, which means that the variable fee paid by the merchant to the card issuer is bigger than the benefits he receives from that particular electronic payment method. Cash payments do not produce any benefits.

Consumers also receive net benefits from paying by card, $b_{p}$, but no benefits from cash payments. Here, the benefits may arise from the delayed payment, insurance cover or cash-back options. The benefits are the same for all consumers, but could differ across card purveyors. The $\mathbb{D}_{b_{p}}$ is a subset of real numbers and as in the case of the merchants could also include negative values.

Finally, the issuer of the payment method has to decide how much it should spend in publicity $l_{p} \in \mathbb{D}_{l_{p}}$, in order to increase the number of consumers and merchants using the electronic card that he is providing. The publicity domain, $\mathbb{D}_{l_{p}}$, is a subset of real numbers. Thus, the variables controlled by the card purveyors are $F_{p}$, $\Gamma_{p}, \beta_{p}, b_{p}$ and $l_{p}$ and those form its strategy.

${ }^{2}$ We have modelled local network connections.

\subsection{Decisions of the Agents}

\subsubsection{Merchants' Decisions}

After certain period of interactions ${ }^{3}$ at the point of sale the merchants have elements to decide to which new cards to subscribe and which old subscriptions to keep. In order to do so, at the beginning of the simulation, merchants start with certain number of cards assigned to them. Then for each commercial transaction the establishments keep track of the cards presented at the counter. Every time a card $p \in \mathcal{P}$ is presented to the merchant $m \in \mathcal{M}$ and he has a subscription to this card $p \in \mathcal{P}_{m}$ with $\left|\mathcal{P}_{m}\right|=N_{\mathcal{P}_{m}}$ he increases the score of the card $\theta_{m, p}^{-}$ by one. Here, $\theta_{m, p}^{-}$is an element of the vector defined as

$$
\left(\theta_{m, 1}^{-}, \cdots, \theta_{m, N_{P_{m}}}^{-}\right)
$$

On the other hand, if the merchant does not have subscription to the card, i.e. $p \in \mathcal{P}_{m}^{1}$, the score of the card $\theta_{m, p}^{+}$is increased by one, given that $\theta_{m, p}^{+}$is an element of the vector:

$$
\left(\theta_{m, 1}^{+}, \cdots, \theta_{m, N \overline{\mathcal{P}}_{m}}^{+}\right)
$$

The merchant decides to cancel the subscription of a card with probability ${ }^{4}$

$$
\pi_{m, p}^{-}=\frac{x_{m}^{-} q}{x_{m}^{-} q+\exp \left(\frac{\theta_{m, p}^{-}}{\theta_{m}}\right)}
$$

where $\theta_{m}$ denotes the number of cards presented. Similarly he decides to subscribe to a new card with probability

$$
\pi_{m, p}^{+}=\frac{\exp \left(\frac{\theta_{m, p}^{+}}{\theta_{m}}\right)}{x_{m}^{+} q+\exp \left(\frac{\theta_{m, p}^{+}}{\theta_{m}}\right)}
$$

where $x_{m}^{-} q$ and $x_{m}^{+} q$ represent the inertia to add or drop a card; $q=\left(1+\Gamma_{p}+N_{\mathcal{P}_{m}}+\frac{\varepsilon}{\beta_{p}}\right)$, whereas $x_{m}^{-}$and $x_{m}^{+}$are constants.

\subsubsection{Consumers' Decision}

In the model the consumers take three decisions: which merchant to visit, which card to use and similar to the merchants' decision, to which card to subscribe?

\footnotetext{
${ }^{3}$ The number of interactions is dissimilar across merchants and it is determined by Poisson distribution specific for each $m$.

${ }^{4}$ The probabilities defined in Equations (1) and (2) are affected by the publicity applied by each payment card provider.
} 
Regarding the first decision, we assume that when deciding which merchant to visit, the consumer has not yet decided which of the cards he holds will be used. Suppose $\mathcal{P}_{c, m}$ is the set of cards the consumers and merchants have in common. Given that $\left|\mathcal{P}_{c, m}\right|=N_{\mathcal{P}_{c, m}}$, we assume that the more common payment cards the merchant $m$ and the consumer $c$ have, the more attractive a merchant becomes. This is due to the fact that the consumer always carries all his cards with him and he decides which card to use at the moment of the payment. Additionally, the smaller the distance $d_{c, m}$ between the consumer and the merchant, the higher the possibility for this merchant to be chosen by the consumer. From these deliberations we propose to use a preference function for consumer to visit merchant:

$$
v_{c, m}=\frac{\frac{N_{\mathcal{P}_{c, m}}}{d_{c, m}}}{\sum_{m^{\prime} \in \mathcal{M}_{c}} \frac{N_{\mathcal{P}_{c, m^{\prime}}}}{d_{c, m^{\prime}}}}
$$

Each consumer $c \in C$ chooses a merchant $m \in \mathcal{M}$ with probability $v_{c, m}$ as defined in 3 . The consumers will continuously update their beliefs regarding the number of common payment cards for all merchants they may visit. With respect to the second decision, the consumer decides which payment card he wants to use at the counter of the chosen merchant. We assume a preferred card choice, given that the consumer chooses the card with the higher benefits $b_{p}$, alternatively if the merchant does not accept any of the consumers' cards the transaction is settled using cash payment.

Finally, after certain periods of interactions ${ }^{5}$ the cardholders decide which new cards to subscribe to and which old subscriptions to keep.

This decision has the following structure. Similarly to the merchants, initially consumers have certain number of cards $\mathcal{P}_{c}$ with $\left|\mathcal{P}_{c}\right|=N_{\mathcal{P}_{c}}$. Every consumer $c \in C$ keeps track whether the cards he owns are accepted by the merchant or not. If card $p \in \mathcal{P}_{c}$ is accepted by the visited merchant $m \in \mathcal{M}_{c}$, the consumer increases the score of the card $\omega_{c, p}^{-}$by one. Here $\omega_{c, p}^{-}$is an element of the vector specified as

$$
\left(\omega_{c, 1}^{-}, \cdots, \omega_{c, N_{\mathcal{P}_{\mathcal{C}}}}^{-}\right)
$$

Assume that he cancels his subscription with probability ${ }^{6}$ defined in 4 , given that the number of merchants visited is $\omega_{c}$.

\footnotetext{
${ }^{5}$ The number of interactions is different across consumers and is defined by individual Poisson distribution.

${ }^{6}$ The probabilities defined in Equations (4) and (5) are affected by the publicity applied by each payment card provider.
}

$$
\pi_{c, p}^{-}=\frac{x_{c}^{-} k}{x_{c}^{-} k+\exp \left(\frac{x_{c, p}^{-}}{\omega_{c}}\right)}
$$

Here $x_{c}^{-} k$ accounts for the inertia of the consumer to change cards; $k=\left(1+F_{p}+N_{\mathcal{P}_{c}}+\frac{\varepsilon}{b_{p}}\right)$, whereas $\varepsilon$ and $x_{c}^{-}$are constants.

At the other hand, let $\mathcal{P}_{c}^{-}$with $\left|\mathcal{P}_{c}^{-}\right|=N_{\mathcal{P}_{c}^{-}}$be the set of payment cards, to which the consumer does not have subscription. Suppose consumer $c$ visits a merchant $m$ and they are faced with the situation, in which they do not have cards in common. The set of cards the merchant accepts is $\mathcal{P}_{m} \neq\{\varnothing\}$. In that case the consumer increases the score $\omega_{c, p}^{+}$by one $\forall p \in \mathcal{P}_{m} \subset \mathcal{P}_{c}^{-}$. Here $\omega_{c, p}^{+}$is an element of the vector, which is defined as

$$
\left(\omega_{c, 1}^{+}, \cdots, \omega_{c, N_{\mathcal{P}_{\mathcal{C}}}}^{+}\right)
$$

Given that $\omega_{c}^{+}$is a constant, the probability of subscribing to these cards is then determined by

$$
\pi_{c, p}^{+}=\frac{\exp \left(\frac{\omega_{c, p}^{+}}{\omega_{c}}\right)}{\omega_{c}^{+} k+\exp \left(\frac{\omega_{c, p}^{+}}{\omega_{c}}\right)} .
$$

\subsubsection{Payment Cards Providers' Decisions}

The payment card providers' decision is to define what strategy they are going to use. For that reason we define the solution space of the payment card's strategy as

$$
\mathbb{S}=\mathbb{D}_{F_{p}} \times \mathbb{D}_{\Gamma_{p}} \times \mathbb{D}_{b_{p}} \times \mathbb{D}_{\beta_{p}} \times \mathbb{D}_{l_{p}}
$$

rewritten as

$$
\begin{aligned}
& \mathbb{S}=\mathbb{D}_{1} \times \cdots \times \mathbb{D}_{5} \text { with } \\
& \mathbb{D}_{1}=\mathbb{D}_{F p}, \cdots, \mathbb{D}_{5}=\mathbb{D}_{l_{p}} .
\end{aligned}
$$

In addition we assume that the cost of publicity, $l_{p}$, spend by the card issuer in each interaction, has a direct impact in the consumers' and the merchants' decisions to subscribe/cancel a card. The probabilities, $\pi_{m, p}^{-}, \pi_{m, p}^{+}$, $\pi_{c, p}^{-}, \pi_{c, p}^{+}$, defined in Equations (1), (2), (4) and (5) are then adjusted according to the rule presented in the following equation

$$
\Delta \pi=\tau \pi(2-\pi)
$$

Here $\pi$ substitutes any of the above probabilities, $\Delta$ represents the differences between the original value of $\pi$ and the adjusted $\pi$, and finally $\tau=\alpha\left(\varphi-\exp \left(-l_{p}\right)\right)$. The constants $\alpha$ and $\varphi$ satisfy the constrains 
$\pi-\Delta \pi \geq 0$ and $\pi+\Delta \pi \leq 1$.

Let $\boldsymbol{S}=\left(s_{1}, \cdots, s_{N_{\mathcal{P}}}\right)$ be the vector of sample strategies for all payment methods. The decisions of a particular payment card provider are an instantiation of this vector. Sampling $s_{p}$ from $\mathbb{S}$ could follow a random selection process. Alternatively, the vector $\left(s_{1}, \cdots, s_{N_{\mathcal{P}}}\right)$ could be the result of an extensive search over the strategy space constrained by the complex shape of the modelled demand. In the next section we briefly explain how we have applied the GPBIL algorithm, which incorporates positive and negative learning, and is able to approximate versatile distributions.

\section{Applying GPBIL in Finding Profit Maximizing Strategy}

The strategy's domain $\mathbb{D}_{i}$ is interval of real numbers. Assume a probability distribution functions $\mathbb{F}_{\mathbb{D}_{i}}: \mathbb{R} \rightarrow[0,1]$ for unconditional random variables over the ranges $\mathbb{D}_{i}$, we define the joint probability distribution $\mathbb{F}_{\mathbb{S}}$ over $\mathrm{S}$ by

$$
\mathbb{F}_{\mathbb{S}}=\mathbb{F}_{\mathbb{D}_{1}} \cdots \mathbb{F}_{\mathbb{D}_{S}}
$$

All electronic cards providers have the same joint probability distribution and we are using it firstly to sample individual strategies from the space, and secondly to modify $\mathbb{F}_{\mathbb{S}}$ through learning.

We have defined $S=\left(s_{1}, \cdots, s_{N_{p}}\right)$ as the vector of strategies of all payment methods in one execution of the artificial payment card market. Additionally, we define $\phi_{p}=\left(\Phi_{p}, N_{T_{p}^{*}}, p\right)$ as the measurement of the performance achieved in one execution of the model for one payment method. The three elements that compose it are the profit of the card issuer $\Phi_{p}$, the number of transactions obtained in the market $N_{T^{*}}$ and the corresponding index of the card $p$. The vector ${ }^{*}\left(\phi_{1}, \cdots, \phi_{N_{p}}\right)$ represents the performance of all payment cards in one execution of the artificial market, after certain number of interactions among consumers and merchants.

In Figure 1 we present the MARKET-GPBIL process used to find a profit-maximizing strategy. In our application the strategy should fulfill the following main objective: obtain the highest possible profit $\phi_{p}$ under the constraint that the number of transactions obtained $N_{T_{p}^{*}}$ should be equal or above average.

The first step is to initialize the joint probability function. This is performed by the function initialisation, which receives as a parameter the solution space $\mathbb{S}$ and returns the initialized joint probability function, $\mathbb{F}_{\mathbb{S}}$. The main part of the algorithm consists of a loop over $R$ runs. At the beginning of each run every payment card provider selects a strategy $S_{p}$. This process is carried out by the function sampling (line 5 of the GPBIL algorithm Figure 1), which returns a strategy $S_{p}$ for each one of

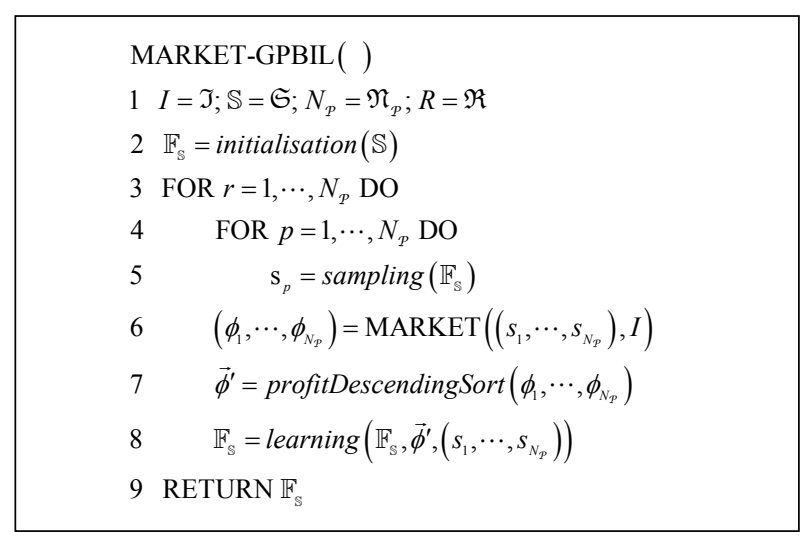

Figure 1. The process MARKET-GPBIL for profit-maximizing strategies.

the payment cards, following the probability distribution function $\mathbb{F}_{\mathbb{S}}$.

Thereafter, in line 6, we instantiate the process MARKET with the strategy vector $\left(s_{1}, \cdots, s_{N_{P}}\right)$ and the number of interactions $I$. This process reproduces the transactions at the point of sale among consumers and merchants. At the end it returns a vector of all payment cards performance measures $\left(\phi_{1}, \cdots, \phi_{N_{\mathcal{P}}}\right)$.

Before the learning function is carried out, the performance of the payment cards providers $\left(\phi_{1}, \cdots, \phi_{N_{p}}\right)$ are sorted (line 7) according to the profit $\Phi_{p}$ achieved at the MARKET. The new vector is denoted $\vec{\phi}^{\prime}$. Following this step, the joint probability function $F_{S}$ is modified by a learning process (line 8). This task is accomplished considering the market share $N_{T_{*}^{*}}$ obtained in the MARKET. More specifically, the function receives as parameters the current values of the joint probability distribution $\mathbb{F}_{\mathbb{S}}$, the profit ordered according the performance $\vec{\phi}^{\prime}$ and the vector of strategies $\left(s_{1}, \cdots, s_{N_{p}}\right)$. The strategies that have obtained better than or equal to the average number of transactions are considered examples of positive learning, whereas the rest of the strategies form the set of negative learning examples.

Finally, in line 9, the GPBIL algorithm returns the resulting joint probability distribution. This function is used as a probabilistic model to generate strategies that fulfill the main objective: to achieve the highest possible profit, given the additional restriction of obtaining above or equal average number of card transactions. In the next section we compare the performance of randomly generated strategies over the performance of the strategies resulting from the joint probability distribution found by the MARKET-GPBIL.

\section{The Setting of the Experiment}

In this section we present the setting of the model's parameters and the structure of the efficiency test. We have 
defined the number of interactions among consumers and merchants as $I$ and it is settled at $I=3000$; the Poisson distribution, used to determine the decision period of consumers and merchant, has a mean $\lambda=20$. The sets of consumers $C$ and merchants $\mathcal{M}$ are instantiate with $N_{C}=1100$ and $N_{\mathcal{M}}=125$. The rest of the user defined parameters are divided in two tables. In Table 1 we have listed the values of the constants, which impact the decisions of consumers and merchants, whereas in Table 2 the domain of each element of the strategy space is presented. The efficiency test consists of comparing the performance of the profit-maximizing strategy with the performance of randomly generated strategy.

In order to do so, we have settled a market with nine competitors. Using the parameter setting described above, in the simulation eight of the nine competitors use the same profit-maximizing strategy, whereas one competitor applies a randomly generated strategy. We test five of the ten GPBIL-strategies presented in [17]. Each of the five exercises consists in comparing the evolved strategy against ten random strategies in independent executions of the model. We use the same randomly generated strategies in the five comparisons.

In Tables $\mathbf{3}$ and $\mathbf{4}$ the set of random and GPBIL strategies are presented. In the first column of the tables we list the identifier of the strategy ${ }^{7}$, whereas in the rest of the columns we present the strategy's elements.

Finally, we list the measurements used to evaluate the performance of the different kinds of strategies.

- $\Phi_{p}^{g p}$ is the profit of the competitor with the randomly generated strategy;

- $\Phi_{p}^{r m}$ is the average profit of the eight competitors using the strategy obtained by the GPBIL;

- $\Phi_{I_{p}}^{g p}$ is the average number of card transactions of the eight payment card providers, using the strategy obtained by the GPBIL;

- $\Phi_{I_{p}}^{r m}$ is the number of transactions of the competitor, using the randomly generated strategy.

\section{Results}

In this section we present the results obtained from the evaluation of the performance of the GPBIL strategies in maximizing profit and obtaining on average number of transactions. The section is divided in two parts, both of them based on the data shown in Tables $5-9^{8}$.

In the first part in five graphics the individual performance of each GPBIL strategy tested is shown against

\footnotetext{
${ }^{7}$ This number is used latter to relate each strategy to its performance. ${ }^{8}$ The tables are organized in the following way: in the first column the random strategy's identifier is presented, in the second and third columns the number of transactions and the profit of the random strategy are listed, and finally in the last two columns the average number of transactions and the average profit of the profit-maximizing strategy are presented.
}

Table 1. Constants used in the end-users' decisions.

\begin{tabular}{clc}
\hline Symbol & \multicolumn{1}{c}{ Description of the Constants } & Value \\
\hline$\varepsilon$ & constant for the inertia to changes & 1 \\
$x_{C}^{-}$ & accounts for the consumers' inertia to drop cards & 0.005 \\
$x_{C}^{+}$ & accounts for the consumers' inertia to add cards & 2 \\
$x_{m}^{-}$ & accounts for the merchant' inertia to drop cards & 0.05 \\
$x_{m}^{+}$ & accounts for the merchant' inertia to add cards & 9 \\
$\alpha$ & accounts for the impact of the publicity cost & 0.1 \\
$\varphi$ & account for the impact of the publicity cost & 5 \\
\hline
\end{tabular}

Table 2. Strategy's domains.

\begin{tabular}{clc}
\hline Symbol & \multicolumn{1}{c}{ Domain } & Value \\
\hline $\mathbb{D}_{F_{p}}$ & Consumers Fixed Fee Domain & {$[0 ; 10]$} \\
$\mathbb{D}_{\Gamma_{p}}$ & Merchants Fixed Fee Domain & {$[0 ; 10]$} \\
$\mathbb{D}_{b_{p}}$ & Domain of Consumers' Benefits & {$[-1 ; 1]$} \\
$\mathbb{D}_{\beta_{p}}$ & Domain of Merchants' Benefits & {$[-1 ; 1]$} \\
$\mathbb{D}_{l_{p}}$ & Publicity Cost's Domain & {$[0 ; \infty]$} \\
\hline
\end{tabular}

Table 3. Randomly generated strategies.

\begin{tabular}{cccccc}
\hline Id & $F_{P}$ & $\Gamma_{P}$ & $\beta_{P}$ & $b_{P}$ & $l_{P}$ \\
\hline 1 & 5.50 & 0.49 & -0.10 & -0.79 & 14.84 \\
2 & 5.82 & 0.42 & -0.74 & 0.36 & 14.24 \\
3 & 6.62 & 3.96 & 0.12 & -0.25 & 0.70 \\
4 & 0.92 & 4.49 & -0.66 & -0.09 & 5.68 \\
5 & 6.07 & 6.17 & -0.87 & 0.04 & 15.42 \\
6 & 3.16 & 3.95 & -0.66 & -0.88 & 0.69 \\
7 & 6.16 & 1.87 & 0.48 & -0.72 & 1.92 \\
8 & 7.80 & 5.87 & -0.15 & -0.64 & 0.51 \\
9 & 6.31 & 3.57 & -0.07 & 0.53 & 8.29 \\
10 & 6.57 & 3.93 & 0.56 & -0.47 & 12.80 \\
\hline
\end{tabular}

Table 4. Profit-maximizing strategies.

\begin{tabular}{cccccc}
\hline $\mathrm{Id}$ & $F_{P}$ & $\Gamma_{P}$ & $\beta_{P}$ & $b_{P}$ & $l_{p}$ \\
\hline 1 & 7.57 & 0.00 & -1.00 & -1.00 & 11.71 \\
2 & 5.66 & 0.00 & -1.00 & -1.00 & 10.82 \\
3 & 5.33 & 0.00 & -1.00 & -1.00 & 7.66 \\
4 & 6.03 & 0.00 & 0.48 & -1.00 & 11.82 \\
5 & 3.51 & 0.00 & 1.00 & -1.00 & 11.81 \\
\hline
\end{tabular}


Table 5. Efficiency test, strategy 1.

\begin{tabular}{ccccc}
\hline Id & $N_{I_{p}}^{m m}$ & $\Phi_{p}^{r m}$ & $N_{I_{p}}^{g p}$ & $\Phi_{p}^{g p}$ \\
\hline 1 & 293587 & 4183603 & 190045 & 6112638 \\
2 & 155146 & 4777710 & 196131 & 6074918 \\
3 & 71466 & 3818894 & 205027 & 6127442 \\
4 & 109469 & 1267441 & 199041 & 6016778 \\
5 & 31243 & 5217924 & 209679 & 6148302 \\
6 & 154401 & 3352744 & 197677 & 6045649 \\
7 & 190495 & 4020676 & 195060 & 6062770 \\
8 & 63695 & 3896739 & 206835 & 6154275 \\
9 & 59307 & 3726206 & 205612 & 6053948 \\
10 & 56643 & 4061625 & 208539 & 6177387 \\
\hline
\end{tabular}

Table 6. Efficiency test, strategy 2.

\begin{tabular}{ccccc}
\hline Id & $N_{I_{p}}^{m m}$ & $\Phi_{p}^{m m}$ & $N_{I_{p}}^{g p}$ & $\Phi_{p}^{\mathrm{gp} p}$ \\
\hline 1 & 270086 & 4028220 & 200859 & 5508231 \\
2 & 132753 & 4580050 & 206786 & 5467251 \\
3 & 65463 & 3638983 & 215090 & 5531546 \\
4 & 89048 & 1195634 & 211330 & 5404561 \\
5 & 25704 & 4932694 & 220308 & 5537538 \\
6 & 138006 & 3235442 & 208256 & 5429815 \\
7 & 173742 & 3837502 & 206364 & 5482818 \\
8 & 56985 & 3745939 & 217497 & 5532962 \\
9 & 53913 & 3525837 & 215560 & 5458349 \\
10 & 51820 & 3860624 & 218343 & 5554859 \\
\hline
\end{tabular}

Table 7. Efficiency test, strategy 3.

\begin{tabular}{ccccc}
\hline Id & $N_{I_{p}}^{m m}$ & $\Phi_{p}^{m}$ & $N_{I_{p}}^{g p}$ & $\Phi_{p}^{g p}$ \\
\hline 1 & 264663 & 3990413 & 202948 & 5367858 \\
2 & 130425 & 4586414 & 208333 & 5328684 \\
3 & 64575 & 3603082 & 216486 & 5394379 \\
4 & 87680 & 1187830 & 212724 & 5271941 \\
5 & 24624 & 4897958 & 222170 & 5389579 \\
6 & 133747 & 3193190 & 210157 & 5291645 \\
7 & 173181 & 3793127 & 207436 & 5346346 \\
8 & 54461 & 3710916 & 219947 & 5399237 \\
9 & 53267 & 3511761 & 217211 & 5342348 \\
10 & 50646 & 3832682 & 220048 & 5412604 \\
\hline
\end{tabular}

Table 8. Efficiency test, strategy 4.

\begin{tabular}{ccccc}
\hline Id & $N_{I_{p}}^{m m}$ & $\Phi_{p}^{r m}$ & $N_{I_{p}}^{g p}$ & $\Phi_{p}^{g p}$ \\
\hline 1 & 158276 & 4051594 & 242115 & 4379904 \\
2 & 120743 & 4864813 & 245621 & 4375830 \\
3 & 43607 & 3837620 & 249250 & 4415086 \\
4 & 70769 & 1225230 & 240926 & 4291554 \\
5 & 27929 & 5252087 & 251869 & 4384489 \\
6 & 99064 & 3228579 & 244468 & 4357959 \\
7 & 129401 & 4051465 & 241096 & 4375036 \\
8 & 32625 & 3875549 & 250768 & 4459407 \\
9 & 34440 & 3765368 & 250344 & 4412800 \\
10 & 45266 & 4122748 & 247065 & 4399842 \\
\hline
\end{tabular}

Table 9. Efficiency test, strategy 5.

\begin{tabular}{ccccc}
\hline Id & $N_{I_{p}}^{r m}$ & $\Phi_{p}^{r m}$ & $N_{I_{p}}^{g p}$ & $\Phi_{p}^{g p}$ \\
\hline 1 & 115092 & 3711873 & 276873 & 3235839 \\
2 & 78536 & 4502427 & 281802 & 3226917 \\
3 & 31653 & 3539388 & 285434 & 3273227 \\
4 & 47468 & 1130966 & 277161 & 3172209 \\
5 & 17945 & 4842936 & 286763 & 3231322 \\
6 & 69554 & 3037368 & 279898 & 3224163 \\
7 & 73756 & 3711376 & 280569 & 3242955 \\
8 & 23724 & 3669594 & 285970 & 3291332 \\
9 & 21821 & 3476859 & 286019 & 3264873 \\
10 & 27007 & 3807948 & 283580 & 3256304 \\
\hline
\end{tabular}

the ten random strategies used, whereas in the second part we compare the performance between the set of GPBIL strategies against the set of random strategies.

\subsection{Individual Performance}

In this subsection we make an individual comparison between each of the five profit-maximizing strategy (see Figures 2-6) versus the performances of the ten randomly generated strategies ${ }^{9}$. We present the performance of the strategies in two dimensions: profit and number of card transactions.

Observation 1: In the first three exercises the GPBILstrategies (Figures 2-4) have achieved better profit then the ten randomly generated strategies. Further, the evolved strategies report a higher number of card transactions in night of the ten cases tested, i.e. only one randomly ${ }^{9}$ Please refer to Table 3. 


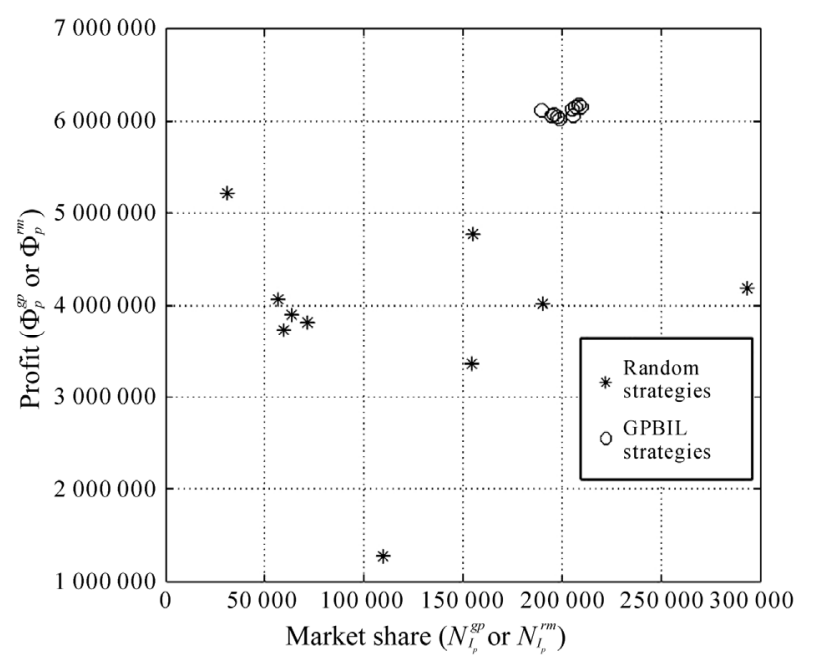

Figure 2. Efficiency test, strategy 1.

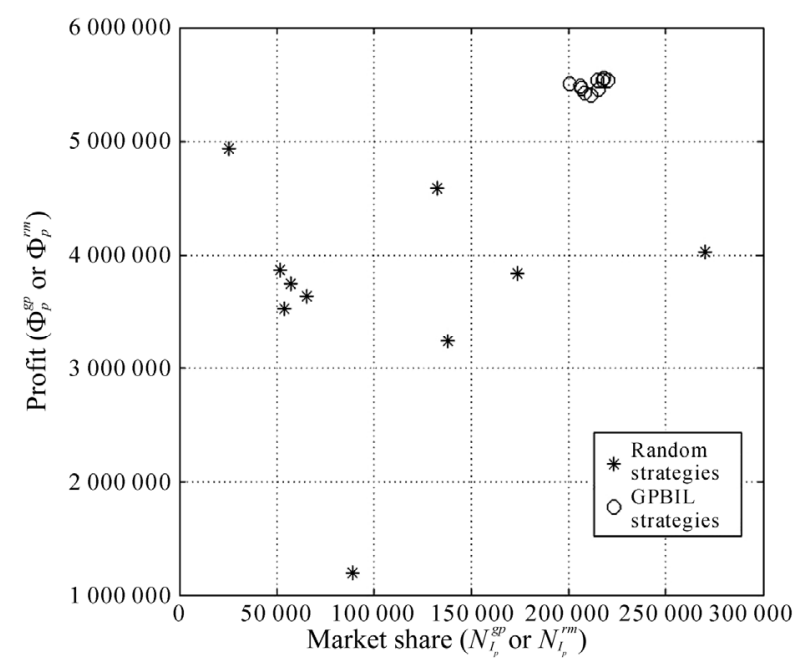

Figure 3. Efficiency test, strategy 2.

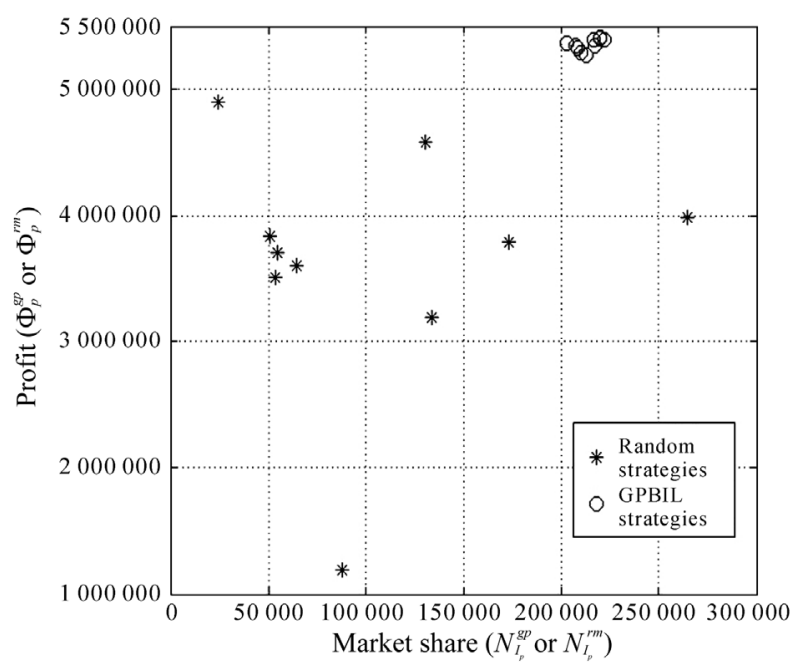

Figure 4. Efficiency test, strategy 3.

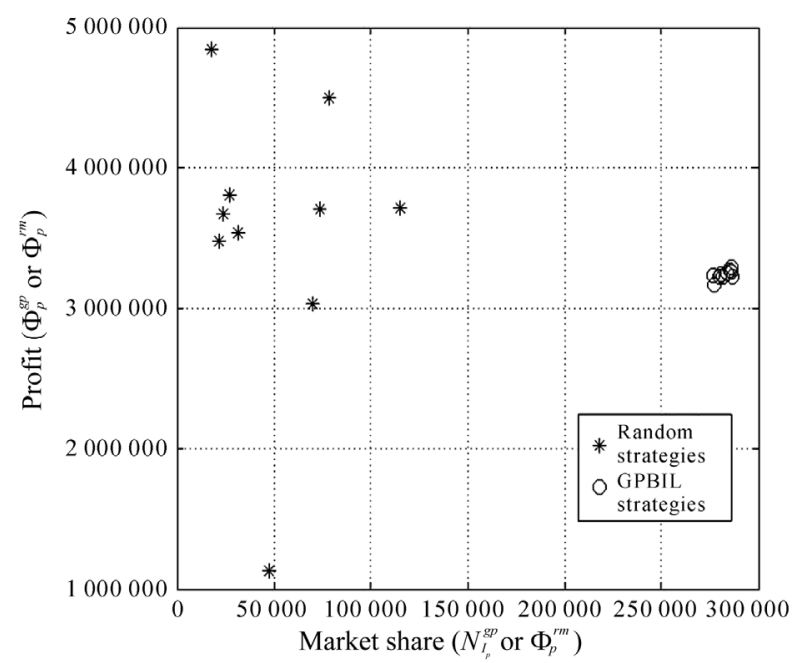

Figure 5. Efficiency test, strategy 4.

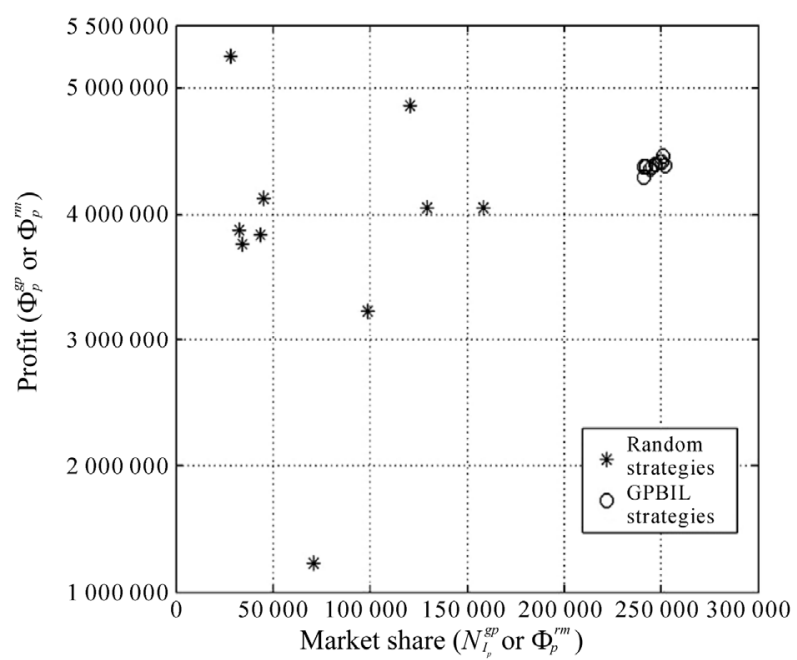

Figure 6. Efficiency test, strategy 5.

generated strategy outrange the three evolved strategies in terms of card transactions, but regarding the profit none of the random strategy could perform better than the GPBIL-strategies 1, 2, and 3. Following, strategy 4 outperforms eight of the ten randomly generated strategies in terms of profit and overpasses all of them in terms of number of card transactions.

Finally, strategy 5 outranges significantly the random strategies in terms of number of card transactions, but in terms of profit was passed in eight of the ten cases tested. Over all, with respect to the profit achieved, the GPBILstrategies have performed statistically better than the randomly generated strategies. Even more, regarding the number of card transactions reported, the performance of the evolved strategies are better than the randomly generated strategies. 


\subsection{The Performance of the Set of GPBIL Strategies vs. the Set of Random Strategies}

In this subsection we present a second comparison, which is made using the same data presented in Tables 5-9. This time we compare on one hand the performance of each GPBIL-strategy among its own records achieved in the ten cases tested and on the other hand the performance of each random generated strategy among its own achievements in the five exercises presented. To that end the performance of each strategy is presented graphically as an area, by plotting together the different performances of the same strategy (either random or GPBIL) resulting from independent instantiations of the model.

More specifically, given that there are five GPBIL strategies tested against ten random strategies, each random strategy has five different performances, whereas each GPBIL strategy has ten performances. In this presentation, if the resulting strategy's area has similar to a rectangle shape, it means that its achievements are similar among the different scenarios and its performance is consistent. On contrary, if the resulting strategy's area has an irregular shape, it means that the performance of the strategy varies among scenarios and it is not consistent.
In Figures $\mathbf{7}$ and $\mathbf{8}$ we plot the random strategies' and GPBIL strategies' areas, resulting from the performance in terms of number of transactions. Next, in Figures $\mathbf{9}$ and $\mathbf{1 0}$ we present the random strategies' and GPBIL strategies' areas formed according to the obtained profit.

Observation 2: In the second comparison, we observe that the areas formed by the individual performance of profit-maximizing strategies have regular shape in contrast to the areas from by the individual performance of the randomly generated strategies, which is irregular. In particular the evolved strategies' areas in terms of profit and number of card transactions are approaching a rectangular shape, whereas the individual areas of the random strategies are irregular in the both dimensions. Further, a more detailed observation which compare the degree of heterogeneity among the sizes of the areas formed by the individual performance inside the same set, allow us to say that the evolved strategies present similar sizes, whereas the randomly generated strategies have significant differences among the individual performances achieved. This observation is true for the both dimensions studied: profit and card transactions.

Observation 3: Regarding the GPBIL-strategies, in Figures $\mathbf{8}$ and $\mathbf{1 0}$ an inverse relation is observed between profit and number of card transactions, i.e. the strategies that have achieved the highest profit are the strategies

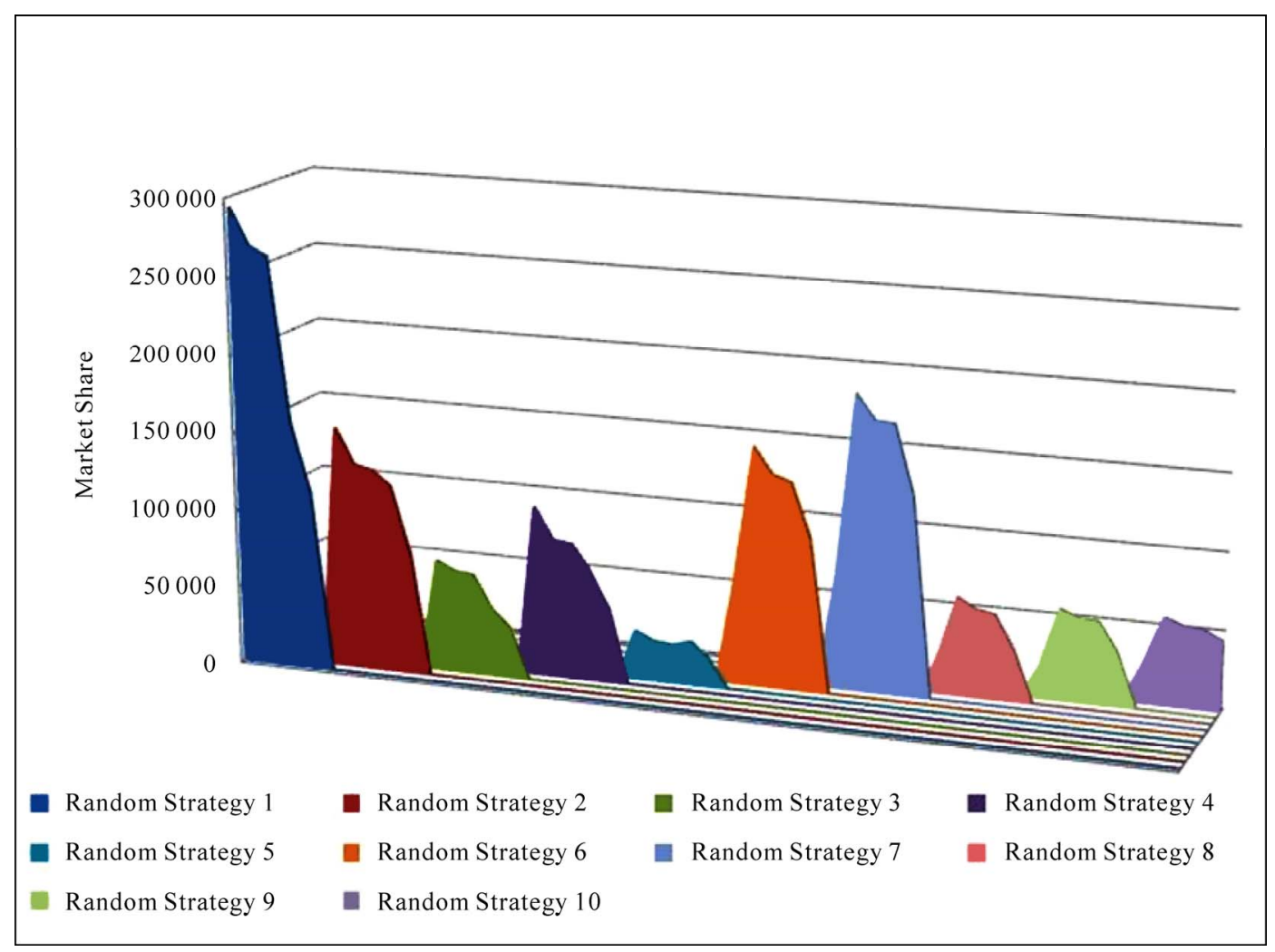

Figure 7. Random strategies performance in terms of number of transactions. 


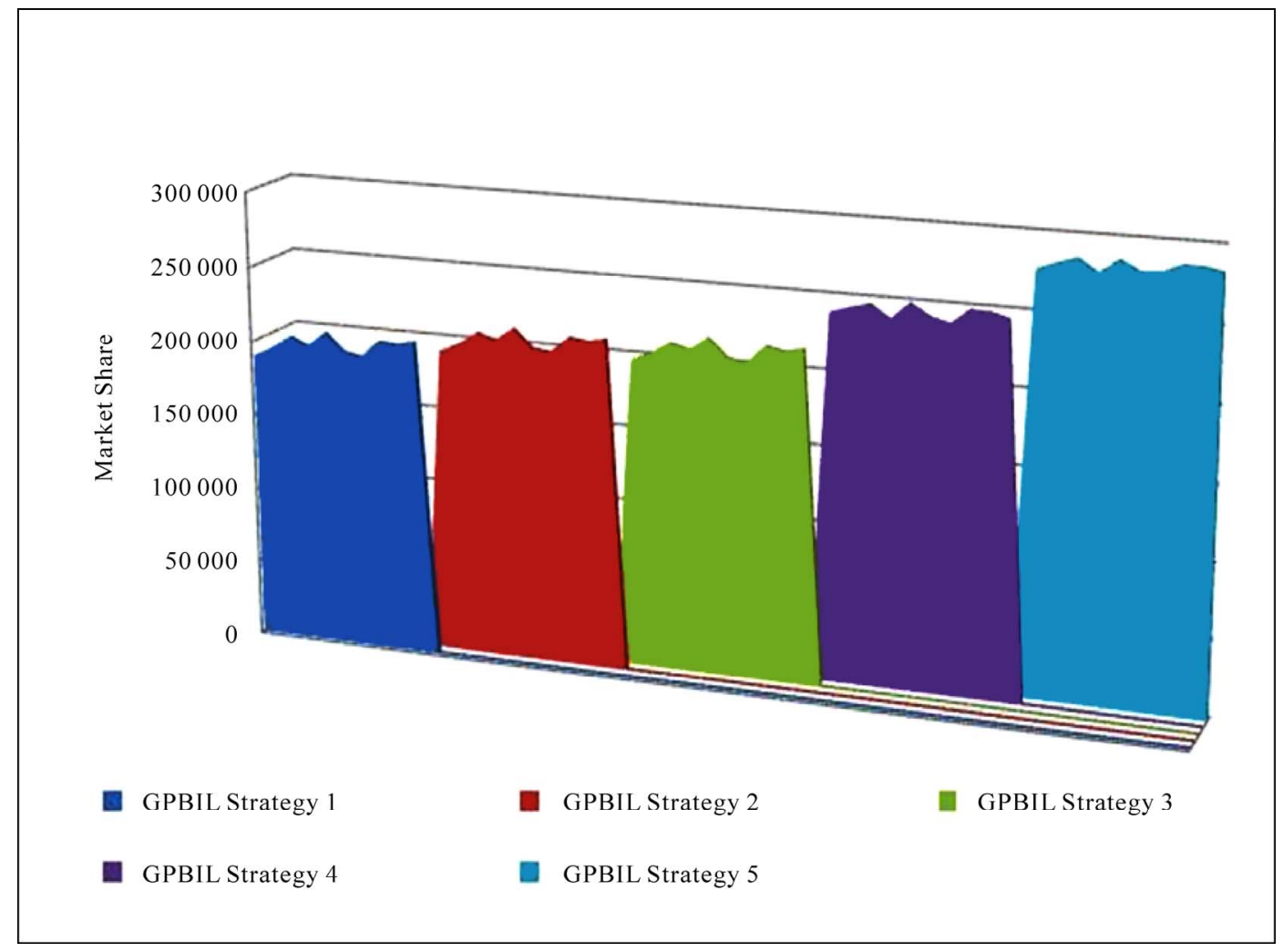

Figure 8. GPBIL strategies performance in terms of number of transactions.

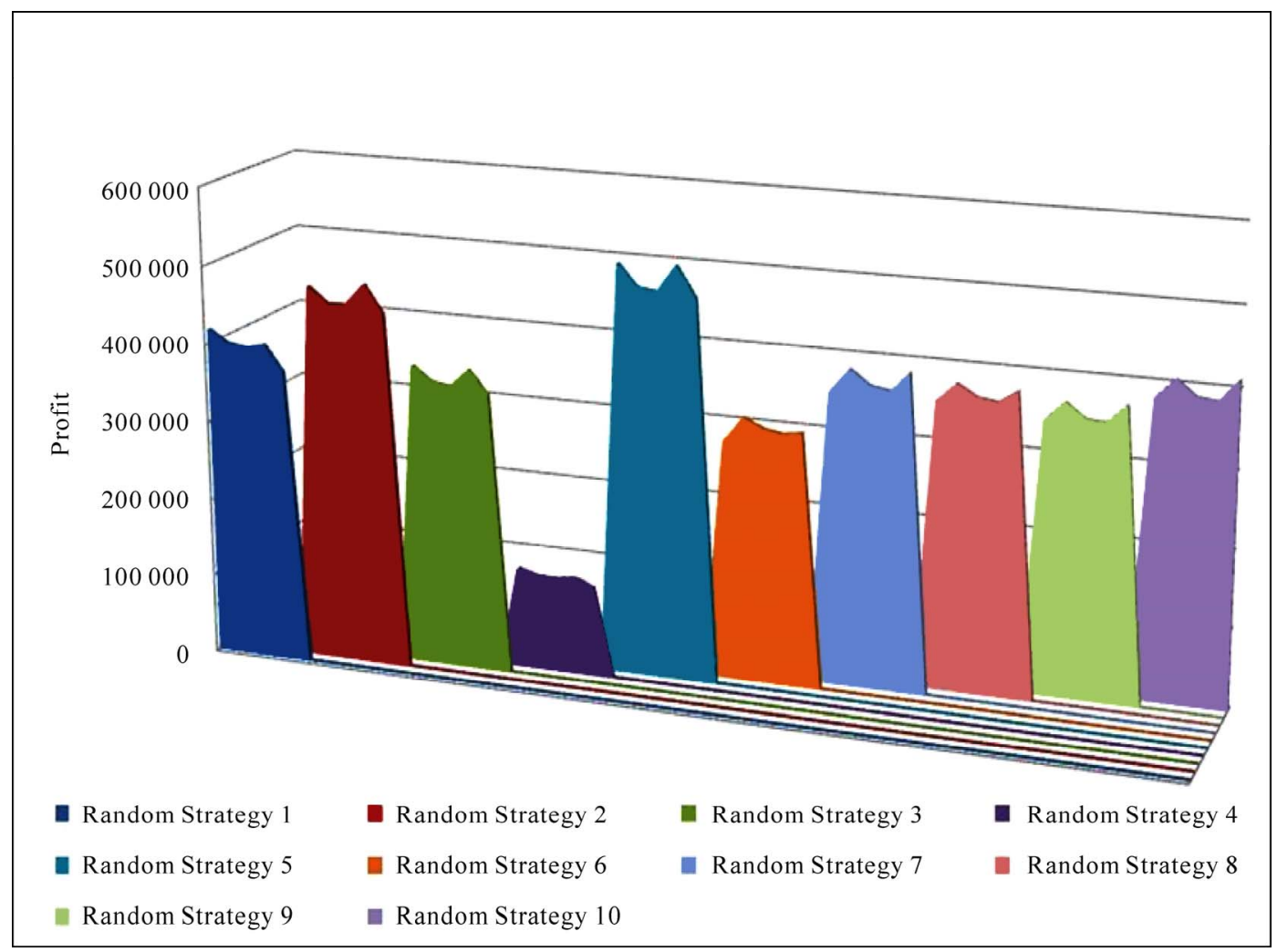

Figure 9. Random strategies performance in terms of profit. 


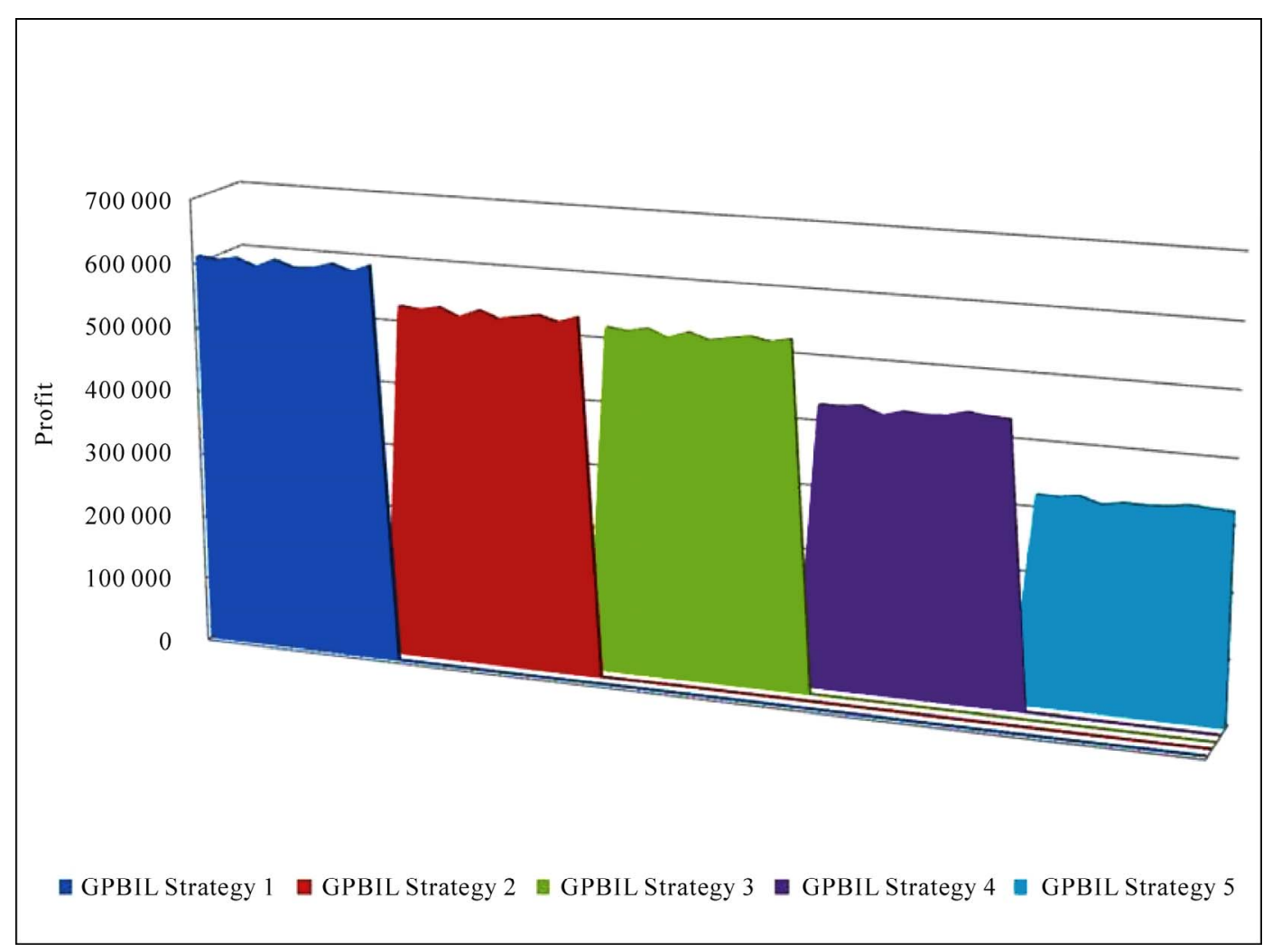

Figure 10. GPBIL strategies performance in terms of profit.

with the lower number of card transactions and vice versa. On the other hand, with respect to the randomly generated strategies the relation between profit and market share observed in Figures 7 and 9 is different. For instance, random strategy 5 , which is the strategy with the highest profit, is also the strategies with the lower number of card transactions, nevertheless random strategy 4 , which had achieved the lower profit is not among the three best performance in terms of card transactions.

Conclusions: from Observations 1, 2 and 3 it follows that the strategies found by the Generalised Population Based Incremental Learning algorithm are efficient strategies in achieving average number of card transactions and maximum profit.

It also follows that with randomly generated strategies is not possible to archive similar performance, therefore the GPBIL-strategies fulfill the purpose they have been designed for.

\section{Conclusions}

In this section we present general conclusions and we give suggestions for further research. The payment card market is characterized with complex relationships among market participants. The two-sided nature of the market gives rise to network externalities that influences the dynamics of the competition. Therefore in the context of the growing importance of the payment cards, the models of the market aimed to study the competition need to incorporate more realistic features. In this sense we consider that the use of agent-based models will allow us to gain better understanding of the payment card market dynamics. In the current study, given the complex shape of the aggregated end-users' demand for electronic payment methods modelled explicitly at the artificial market, the GPBIL algorithm applied has been able to find a price structure and price level that maximize the profit of the card purveyors and has successfully fulfill the objectives of the search. More the all so, we conclude that applying evolutionary techniques, as the GPBIL, in studying relevant aspects of this market opens new research opportunities, untractable with the analytical models. A possible extension of the model is to convert it to a four party scheme, as Visa and Mastercard organized their business this way. From our observations of the market, we believe that the issuers' and acquirers' decisions affect substantially the market competition. Therefore incorporating into the model the sets of issuers and acquirers and their corresponding decisions will allow us to study which rules governing the participants' behavior, could make the market more efficient. Furthermore, of particular interest could be to understand what conditions are required in order for the competitors to offer better 
prices to consumers and merchants.

\section{Acknowledgements}

The authors want to express their gratitude to Francisco Solís and Sara Castellanos for the multiple insights on this and other payment systems matters. The views expressed in this paper are those of the authors and do not involve the responsibility of the institutions they belong to.

\section{REFERENCES}

[1] J.-C. Rochet and J. Tirole, "Cooperation among Competitors: Some Economics of Payment Card Associations," The RAND Journal of Economics, Vol. 33, No. 4, Winter 2002, pp. 1-22.

[2] R. Schmalensee, "Payment Systems and Interchange Fees," Journal of Industrial Economics, Vol. 50, No.2, 2002, pp. 103-122. doi:10.1111/1467-6451.00170

[3] D. Cruichshank, "Competition in UK Banking," Report to the Chancellor of the Exchequer, 2000. Internet Available: http://www.bankreview.org.uk

[4] Reserve Bank of Australia, "Reform of Credit Card Schemes in Australia IV: Final Reforms and Regulation Impact Statement," August 2002.

[5] D. Evans and R. Schmalensee, "The Industrial Organization of Markets with Two-Sided Platforms," National Bureau of Economic Research, September 2005. Internet Available: http://www.nber.org/papers/W11603

[6] J.-C. Rochet, "The Theory of Interchange Fees: A Synthesis of Recent Contributions," Review of Network Economics, Vol. 2, No. 2, June 2003, pp. 97-124. doi:10.2202/1446-9022.1021

[7] D. Evans and R. Schmalensee, "The Economics of Interchange Fees and their Regulation: An Overview," Working Paper 4548-05, Massachusetts Institute of Technology, Sloan, 2005.

[8] J. Wright, "Pricing in Debit and Credit Card Schemes," Economics Letters, Vol. 80, No. 3, 2003, pp. 305-309. doi:10.1016/S0165-1765(03)00143-5

[9] S. Chakravorti and W. R. Emmons, "Who Pays for Credit Cards?" Journal of Consumer Affairs, Vol. 37, No. 2, 2003, pp. 208-230. doi:10.1111/j.1745-6606.2003.tb00451.x

[10] S. M. Markose and Y. J. Loke, "Network Effects on Cash-Card Substitution in Transactions and Low Interest Rate Regimes," The Economic Journal, Vol. 113, No.
487, April 2003, pp. 456-476. doi:10.1111/1468-0297.00124

[11] J.-C. Rochet and J. Tirole, "Platform Competition in Two-Sided Markets," Journal of the European Economic Association, Vol. 1, No. 4, 2003, pp. 990-1029. doi: $10.1162 / 154247603322493212$

[12] G. Guthrie and J. Wright, "Competing Payment Schemes," National University of Singapore Department of Economics, Working Paper 0311, 2003.

[13] S. Chakravorti and R. Roson, "Platform Competition in Two-Sided Markets: The Case of Payment Networks," Working Paper WP-04-09, Federal Reserve Bank of Chicago, Chicago, 2005.

[14] W. Bolt and K. Soramaki, "Competition, Bargaining Power and Pricing in Two-Sided Markets," DNB Working Paper 181, De Nederlandsche Bank, Amsterdam, 2008.

[15] B. Alexandrova-Kabadjova, A. Krause and E. Tsang, "An Agent-Based Model of Interactions in the Payment Card Market," Proceedings of the 8th International Conference on Intelligent Data Engineering and Automated Learning, 2007.

[16] B. Alexandrova-Kabadjova, E. Tsang and A. Krause, "Market Structure and Information in the Payment Card Market," International Journal of Automation and Computing, Forthcoming, 2011.

[17] B. Alexandrova-Kabadjova, E. Tsang and A. Krause, "Evolutionary Learning of the Optimal Pricing Strategy in an Artificial Payment Card Market," Natural Computing in Computational Economics and Finance, Series Studies in Computational Intelligence, Vol. 100, 2008, pp. 233-251.

[18] B. Alexandrova-Kabadjova, E. Tsang and A. Krause, "Competition is Bad for Consumers: Analysis of an Artificial Payment Card Market," Journal of Advanced Computational Intelligence and Intelligent Informatics, Vol. 2, No. 15, 2011, pp. 188-196.

[19] M. Kern, "Parameter Adaptation in Heuristic Search, A Population-Based Approach," Ph.D. Dissertation, University of Essex, Colchester, 2005.

[20] G. E. P. Box and N. R. Draper, "Empirical ModelBuilding and Response Surface," John Wiley \& Sons, Inc., New York, 1986.

[21] E. P. K. Tsang, "Computational Intelligence Determines Effective Rationality," International Journal on Automation and Control, Vol. 5, No. 1, 2008, pp. 63-66. doi:10.1007/s11633-008-0063-6 